\section{Confocal Microscopy for Diagnostic Cytology}
Mathilde E. Boon (1) and Lambrecht P. Kok (2)
(1) Leiden Cytology and Pathology Laboratory (2) Neuro Imaging Center, University of Groningen, The Netherlands l.p.kok@phys.rug.nl

\section{Introduction}

Laser scanning confocal microscopy has been used to analyze cytological specimens for research purposes. However, for many pathologists, its application in diagnostic cytology still is terra incognita. In our laboratory, confocal microscopy has been used for seven years, mainly to solve problems in cervical cytology, in breast aspirates, and for endometrium cytology.

In contrast to histology where we can cut new sections from the block, the cytologic sample is often limited to one smear. Accordingly, when we wish to expand our diagnostic possibilities, we have to extract the desired additional information from that single smear. This is possible by first de-staining the original smear and subsequently re-staining it. In this article, we describe how we restain old slides with the fluorescent dyes Ethidium Bromide and Eosin, a DNA/protein stain, resulting in staining patterns comparable to the diagnostic Papanicolaou and Giemsa methods in that there is an optimal distinction between nucleus and cytoplasm. The DNA

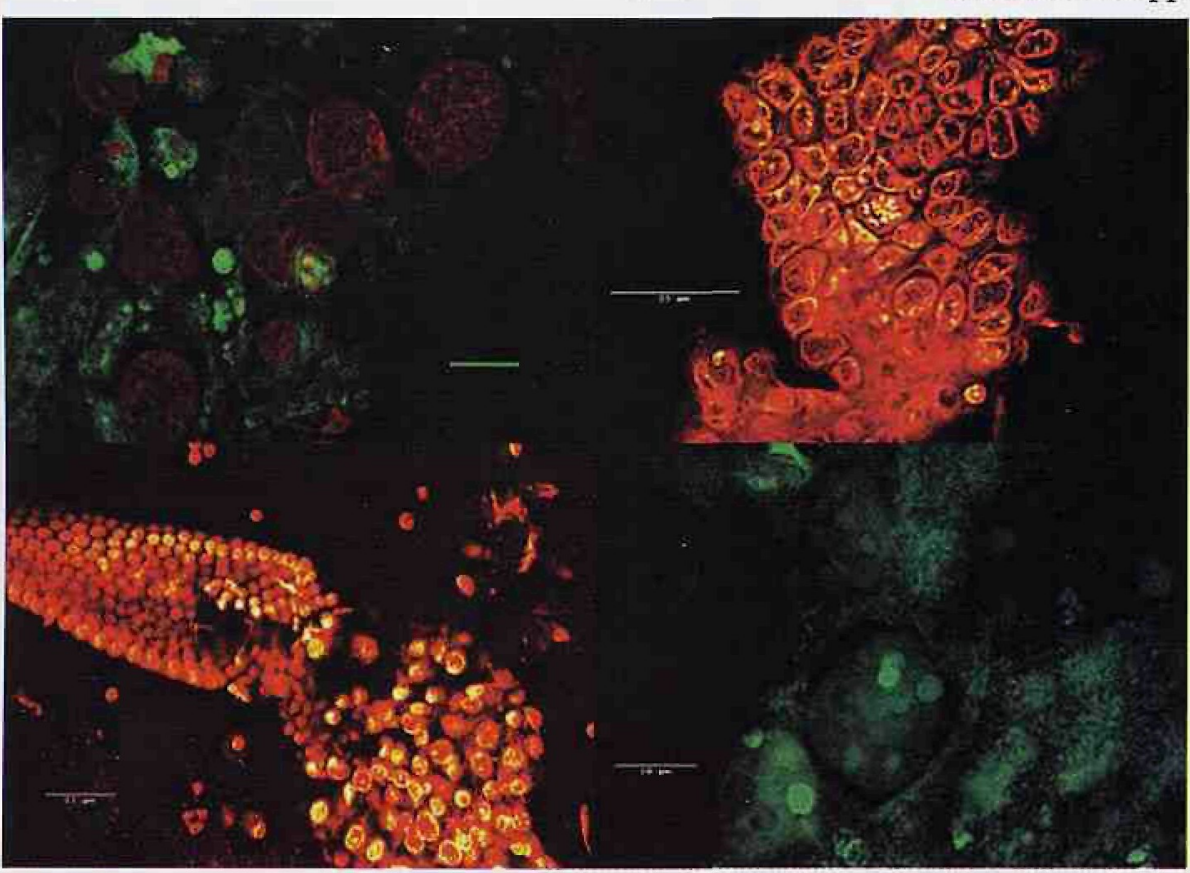

Fig. I Optical thin section of a thick epithelial fragment in a cervical smear, merged images of PMT1 and 2. The patient had a poorly differentiated squamous cell carcinoma. In the original Papanicolaoustained smear, the chromatin was completely blutred. In this confocal image, the fine details of the malignant nuclei are highlighted. Scale bar $=10$ un

Fig. la Optical thin section of an epithelial fragment in an endometrial smear, PMT1 (DNA). We can observe a direct transition for cystic hyperplasia with small nuclei (left) into frank carcinoma with large nuclei containing prominent nucleoli. Scale bar $=25 \mathrm{um}$

Fig. Ib Several Entamoeba Gingivales attached to Actinomyces, found in a cervical smear, PMTI (DNA). This degree of detail of the Attinomyces is not visible in the Papanicolaou-stained smear. The relationship of the two microorganisms is hightighted in this thin confocal section. Scale bar $=25 \mathrm{um}$

Fig. 1c Three-dimensional reconstruction made from serial confocal sections derived from the microorganism Leptothrix with attached coccoid bacteria in a cervical smear PMTI (DNA). Here, confocal microscopy approaches scamning electron microscopy in terms of the information it provides. Scale bar $=5 u m$ of the nucleus (Ethidium Bromide) results in a red fluorescence, the protein in the cytoplasm (Eosin) in a green one.

Both wet-fixed Papanicolaou-stained, and air-dried Giemsastained smears can be used for confocal microscopy. The preexistent ar to distilled water, then immersing in acid alcohol for $20 \mathrm{~min}$ lowed by 20 minutes in $1 \mathrm{~N} \mathrm{HCl}$. The specimens are rinsed

The procedure for the DNA/Protein stain is as follows:

. Place the dehydrated smear for $1 \mathrm{~min}$ in $1 \%$ Eosin $\mathrm{Y}$ (in dis-

.

3. Stain for $5 \mathrm{~min}$ in $0.01 \%$ Ethidium Bromide, Merck, Prod. No. $15(\mathrm{pH}=5.7)$

Rinse in distilled water

36086.

All images were originally produced using the BioRad MRCfocal imaging system equipped with a Krypton/Argonoil, (160-017 Nikon) with the K1 and K2 filter blocks in the scan head. This resulted in the signal from Ethidium Bromide (DNA, red aring in PMT1 and that for Eosin (protein, green ages, the signals from PMTs 1 and 2 were merged. For the three-dimensional reconstructions, exclusively PMT1 images were used. The image data were stored on a floppy disk.

\section{Results}

First, when we try to look into thick epithelial fragments in the smear by conventional means, out-of-focus flare leads to blurred images, lacking in useful detail. However, using confocal microscopy, 'optical sectioning' with the laser beam, produces a 'thin section' from the material on the glass slide, in a similar way to cutting a paraffin block with a microtome. Consequently, in these otherwise imperfect images we are now able to visualize chromatin patterns in great detail (Fig. 1). In addition, (due to the thin sectioning), we can detect mitotic figures (Fig. 2) and glandular lumina in the central part of the fragment, both of which were invisible without confocal microscopy. In fragments from endometrial samples, we can visualize the transition from cystic hyperplasia to adenocarcinoma (Fig. 1a).

Secondly, we can use confocal microscopy to define the bacterial flora in the cervical smear Here, the high resolution of the system allows dependable recognition of the shape and form of these small organisms, even allowing quantitative measurements to be made. In these thin sections, we can visualize the interrelationship of microorganisms such as Actinomyces and Entamoeba Gingivalis in the smear (Fig. 1b). In these confocal images, the fine details of the microorganism structure approach a level hitherto 
unknown to the practising pathologist. In the serial sections, we can see whether the microorganism has actually invaded the cell or is merely sitting on the membrane, (which is impossible to see in the Papanicolaou-stained specimen).

Thirdly, we can gain a great deal of information from the threedimensional reconstruction of the architecture of microorganisms and cells present in the smear and visualize the surface properties in a similar way to that which we encounter in Scanning Electron Microscopy (Fig. 1c).

\section{Discussion}

In cytodiagnosis, analysis of the nuclear pattern is a key issue. The nuclear images in abnormal epithelial fragments, as visualized in confocal microscopy, closely resemble those of plastic-embedded samples. Due to the absence of out-of-focus information, the chromatin patterns seen are less 'coarse' than those observed by conventional light microscopy of the smear. Because of the many cell layers present in these tissue fragments, conventional microscopy produces visualization problems comparable to those present when dealing with thick histological sections $(e . g .30 \mu \mathrm{m})$. In this case, superposed cell layers that are more or less out of focus give an undesirable blur to the images of the cell layer that is in the plane of focus. Additionally, the packing of nuclei in the epithelial fragments of smears is relatively dense (particularly when the cytoplasm is immature). This is due to the fact that this cytoplasm has become 'deflated' compared with embedded tissue where it remains in its original shape and size through infiltration by plastic or paraffin. By the use of confocal microscopy, there is no blur in the optical sections obtained, and many of these difficulties are overcome.

For the other examples presented in this article, the benefits of the confocal images are mainly due to higher magnification, serial

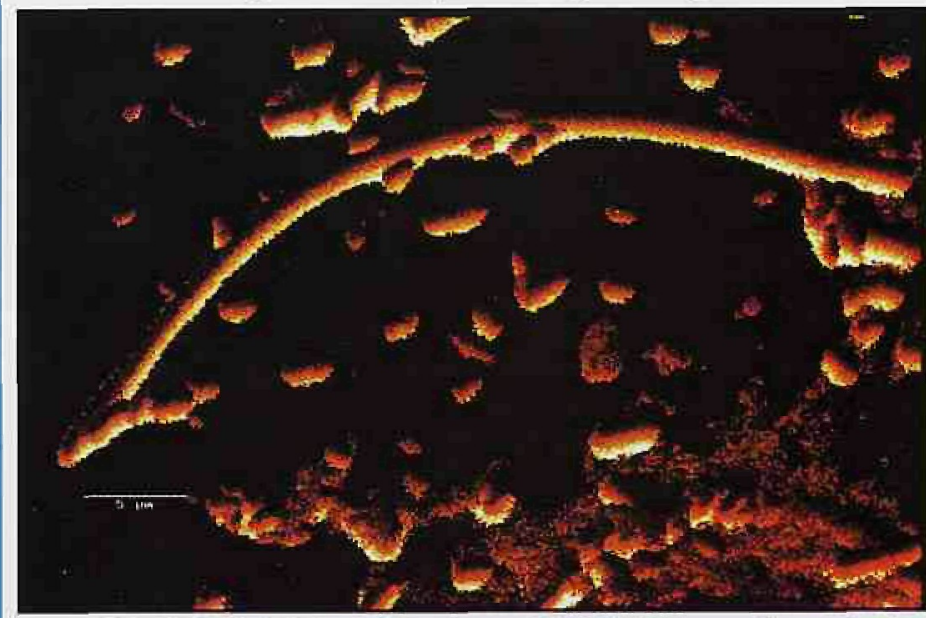

Fig. 2 Optical thin section of an epithelial fragment of a cervical adenocarcinoma in a smear, PMTI (DNA). In the original Papanicolaoustained smear, no details or mitotic figures were visible. The fragment looked solid, devoid of a glandular lumen. In this confocal thin section, each nucleus is visualized and a mitotic figure can be discerned. In the lower part of the section a glandular lumen becomes visible (this became larger in the adjacent serial sections), while in the Papanicolaou-stained smear this epithelial fragment was a solid looking object. Scale bar $=5 \mathrm{um}$

sectioning, and revealing surface characteristics. In serial sectioning, architectural features can be highlighted, such as glandular, tubular or cribriform built from the tumor tissue. Here, confocal cytology comes close to thin serial sectioning in histology. In displaying surface characteristics of cells and microorganisms in cytologic specimens, the scientific quality of confocal imaging approaches that seen with the scanning electron microscope. Seeing these im- ages, the cytologist becomes aware that cytology is very much a three-dimensional rather than two-dimensional study.

\section{Conclusion}

In the cytology handbooks, one does not find photographs of thick epithelial fragments because they are neither photogenic nor diagnostic due to the inherent blur and lack of detail associated with conventional microscopy. However, by using a simple fluorescent DNA/protein stain in combination with confocal microscopy, valuable diagnostic information can be regained from these important, otherwise undiagnosable, smear elements. In this article, we have restricted ourselves to one simple staining method. However, we have learnt that many more staining techniques can be used. To obtain confocal images, fluorescent probes for proteins and viruses can be applied on de-stained smears, and the proliferating cells can be labeled for confocal microscopy with MiB-1. Finally, the confocal digital images can be processed further, for instance by the sophisticated Application Visualization System (AVS) on a CONVEX computer, leading to even finer details than shown in the illustrations.

\section{Acknowledgement}

We wish to thank Ms. Gerda Sutedja-Bol, Ms. Etty Benita, and Ms. Elisabeth Marres for making the images.

This article originally appeared as an Application Note for BioRad Cell Science Division and, together with many others, may be viewed at the company's website http://www.cellscience.bio$\mathrm{rad} . \mathrm{com} / \mathrm{reference} / \mathrm{application}$.htm.

\section{References}

1. BOON ME, KOK LP: Confocal microscopic imaging of the Pap smear. In; Techniques in Diagnostic Pathology, vol. 3, GR Bullock, D van Velzen, MJ Warhol (eds), Academic Press Ltd, London, pp: 77-89, 1992.

2. BOON ME, KOK LP, SUTEDJA G, DUTRIEUX RP: Confocal sectioning of thick, otherwise un-diagnosable cell groupings in cervical smears. Acta Cytol $37: 40-48,1993$

3. BOON ME, SCHUT JJ, BENITA EM, KOK LP: Confocal optical sectioning and three-dimensional reconstruction of carcinoma fragments in Pap smears using sophisticated image data processing. Diagn Cytopathol 10:268-275, 1994.

4. BOON ME, SUTEDJA-BOL G, KOK LP: Confocal microscopy in diagnostic cytology. In: Three-Dimensional Confocal Microscopy: Volume Investigation of Btological Specimens. JK Stevens, LR Mills, JE Trogadis (eds). San Diego, California: Academic Press, Inc. pp 353-375, 1994 .

5. BOON ME, SCHUT JJ, SUURMEIJER AJH, BENITA EM, HUT PKH, KOK LP: Confocal microscopy of false-negative breast aspirates, Diagn Cytopathol 12, $42-50$ (1995)

6. BOON ME, LUZZATTO R, BRUCKER N, RECKTENVALD MS, BENITA EM: Diagnostic efficacy of endometrial cytology with the Abradul Cell Sampler supplemented by laser scanning confocal microscopy. Acta Cytol 40, 277-282 (1996)

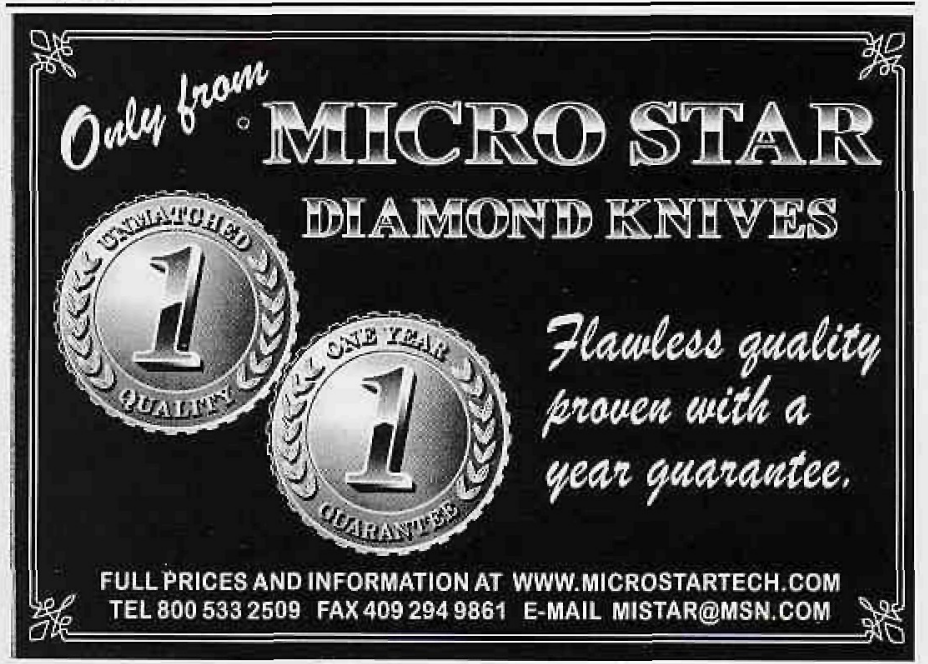

